\title{
Effective computational modeling of erythrocyte electro-deformation
}

\author{
Nicola A. Nodargi · Paolo Bisegna • Federica Caselli
}

Received: date / Accepted: date

\begin{abstract}
Due to its crucial role in pathophysiology, erythrocyte deformability represents a subject of intense experimental and modeling research. Here a computational approach to electro-deformation for erythrocyte mechanical characterization is presented. Strong points of the proposed strategy are: $i$ ) an accurate computation of the mechanical actions induced on the cell by the electric field, ii) a microstructurally-based continuum model of the erythrocyte mechanical behavior, iii) an original rotation-free shell finite element, especially suited to the application in hand. As proved by the numerical results, the developed tool is effective and sound, and can foster the role of electro-deformation in single-cell mechanical phenotyping.
\end{abstract}

Keywords Electro-deformation · Maxwell stress tensor · erythrocyte mechanics · homogenization · finite elements $\cdot$ corotational formulation

\section{Introduction}

The mechanical properties of cells have been shown to be useful markers of cell state by the biophysics community. In particular, there is growing evidence that cell deformability may provide a label-free biomarker for de-

This work was supported by the Scientific Independence of Young Researchers Programme (SIR 2014) under Grant RBSI14TX20-MUSIC "Multidimensional Single-Cell Microfluidic Impedance Cytometry", and by PRIN 2010-11 Programme under Grant 2010BFXRHS_006 "Advanced mechanical modeling of new materials and technologies for the solution of 2020 European challenges".

N. Nodargi · P. Bisegna · F. Caselli

Department of Civil Engineering and Computer Science, University of Rome "Tor Vergata", 00133 Rome, Italy

E-mail: caselli@ing.uniroma2.it termining e.g. metastatic potential, degree of differentiation, or cell activation [16]. Within the cell mechanics literature, the erythrocyte or red blood cell (RBC) is probably the most studied cell type. As discussed in [15], this prominent role is ascribable to the simple structure of the erythrocyte, which is essentially a biconcave liquid capsule enclosed by a membrane, as well as to the importance of RBC deformability in pathophysiology. In fact, erythrocyte deformability is crucial to microvascular function and becomes altered e.g. in malaria, sickle cell anemia, diabete mellitus, and cardiovascular disease [30].

Because of the increased interest in cell mechanics and its relation to pathology, a range of approaches have been developed for single-cell mechanical phenotyping (see, e.g., the reviews $[32,44,1]$ and the references therein). Among them, microfluidic techniques are especially attractive, due to their potential for automated and high-throughput analysis $[28,54]$. In particular, electrically-induced cell deformation in microfluidic systems is receiving increasing attention and has been successfully applied to the mechanical characterization of protoplasts [52], mammalian cells [40], cancer cell lines $[11,29]$, erythrocytes $[20,21]$, and platelets [36].

In order to identify the optimal design of an assay for single-cell mechanical characterization, as well as in order to assist the interpretation of experimental results, an important role is played by modeling and simulation strategies. In particular, in case of electrodeformation systems, they can provide $i$ ) an accurate estimate of the mechanical forces induced on the cell by the electric field, and $i i$ ) the consequent deformation on the basis of the cell mechanical behavior. In [52, 40,21], field-induced actions were estimated by resorting to the Clausius-Mossotti factor under the effective dipole moment assumption, whereas the more general 
Maxwell stress tensor approach [46] was adopted in [11, 36]. In those papers, the actual deformed configuration of the cell was not fully accounted in the computation of field-induced actions. Moreover, simple phenomenological models were adopted to describe the cell mechanical behaviour. In particular, elastic models based on the Young modulus $[11,36]$ or the shear modulus [21], and viscoelastic standard linear solid or power law models [40] were considered.

With respect to erythrocyte mechanical behaviour, refined microstructurally-based constitutive models are available in the literature, taking into account the principal mechanical constituents of the RBC, i.e., the lipid membrane and the underlying spectrin network. Detailed modeling of RBCs at the spectrin level $[18,37]$ is much limited by the high computational cost, and several mesoscopic coarse-grained models have been developed to improve computational efficiency [24]. Microstructurally-based models have been applied to simulate micropipette aspiration [18], optical tweezers experiments [37], and erythrocyte behaviour in shear or Poiseuille flow [24].

In this paper, a computational approach to simulate in silico electro-deformation experiments is presented. In particular, a finite-element based simulation tool is developed, composed of two coupled modules, namely, the electrical module and the mechanical one. The former is devoted to the computation of the mechanical actions induced by the electric field on the RBC (for a given geometry), whereas the latter computes the RBC deformation under a prescribed (electric) load. A fixed-point iteration scheme is adopted to implement the electro-mechanical coupling. This ensures that, at convergence, field-induced actions are relevant to the actual deformed configuration, where equilibrium is enforced.

Some noticeable features of the proposed strategy can be highlighted, that make it unique and effective. In the electrical module, field-induced actions are rigorously computed by means of the Maxwell stress tensor theory (Section 2.1). Moreover, in order to evaluate the electric field distribution (Section 2.2), the cell membrane is treated as a two-dimensional imperfect interface, so that the computational cost is significantly reduced $[2,9,10]$. As a byproduct, an accurate computation of the Maxwell stress tensor is achieved (Section 4.1). In the mechanical module, a microstructurallybased constitutive law is adopted for the cell membrane, along with a simple homogenization procedure (Section 3.2). This way, model parameters have a precise physical meaning, instead of being of phenomenological nature, and at the same time the computational efficiency of a continuum approach is enjoyed. Further- more, in the finite element implementation of the mechanical problem, an original and effective rotation-free shell element is adopted (Section 4.2.2), whose development profitably exploits a corotational formulation (Appendix A).

Careful validation of both modules has been conducted. In particular, the benchmark problem of a coated ellipsoid in a uniform electric field has been considered for the electrical module (Section 5.1). On the other hand, the validation of the mechanical module has been performed by reproducing experiments of RBC deformation by optical tweezers, available in the literature (Section 5.2). The soundness and the efficacy of the proposed coupled approach is shown by means of an application-driven in-silico experiment of erythrocyte electro-deformation (Section 5.3).

The paper is organized as follows. Section 2 and Section 3 describe the electrical and the mechanical module, respectively. Details on the relevant numerical procedures as well as their coupling strategy are provided in Section 4. In-silico experiments are reported in Section 5. Finally, conclusions are drawn.

\section{Electrical module}

A RBC suspended in a fluid is here considered. In presence of an applied electric field, electric charges are induced on the cell membrane, and the interaction of the field with those charges gives rise to mechanical actions responsible for various frequency-dependent behaviours, such as orientation, translation (dielectrophoresis), rotation, and deformation. The purpose of the electrical module is the computation of these mechanical actions.

The investigation of field-induced actions has long been subject of research (see e.g. $[12,47,50,49]$ ) and different strategies have been proposed for their evaluation. Here the Maxwell stress tensor formulation is adopted, which is regarded in the literature as the most general and rigorous approach [51].

\subsection{Mechanical actions induced by the electric field}

As pictured in Figure 1, the electrical domain is modeled as the union of two homogeneous conducting regions $\Omega_{1}$ and $\Omega_{2}$, representing the intracellular space and the suspending fluid, respectively. Their complex conductivities $\sigma_{1}^{*}$ and $\sigma_{2}^{*}$ are given by $\sigma_{k}^{*}=\sigma_{k}+\mathrm{i} \omega \varepsilon_{k} \varepsilon_{\mathrm{v}}$, $k=1,2$, where $\varepsilon_{\mathrm{v}}$ is the vacuum permittivity, and $\sigma_{k}$ and $\varepsilon_{k}$ are the conductivity and relative permittivity of the media, respectively; moreover, $\mathrm{i}$ is the imaginary unit, and $\omega$ denotes the circular frequency. The 


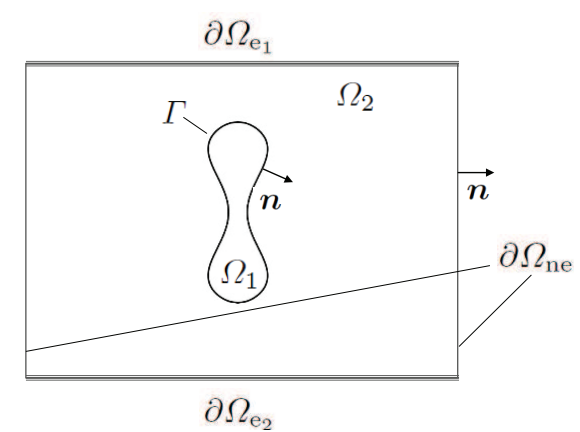

Fig. 1 Domain of the electric problem (schematic 2D representation): $\Omega_{1}$, intracellular space; $\Omega_{2}$ suspending fluid; $\Gamma$, cell membrane; $\partial \Omega_{\mathrm{ne}}$, boundary not covered by electrodes; $\partial \Omega_{\mathrm{e}_{i}}, \boldsymbol{i}$-th electrode; $\boldsymbol{n}$ normal unit vector

cell membrane, which is a thin phospholipid bilayer, is treated as a two-dimensional interface $\Gamma$ with conductance $G$ and capacitance $C$ per unit area, respectively given by the electric conductivity $\sigma_{\mathrm{m}}$ and permittivity $\varepsilon_{\mathrm{m}}$ of the lipid bilayer, divided by its thickness [4]. Therefore, the interface admittance per unit area is $Y=G+\mathrm{i} \omega C$. In the radio-frequency range $Y \approx \mathrm{i} \omega C$, since $G \ll \omega C[26]$, and hence the cell membrane essentially behaves like a capacitor. The finite interface admittance causes the electric potential to jump across the cell membrane [4]. The boundary of the domain is divided into an insulating part $\left(\partial \Omega_{\mathrm{ne}}\right)$, and a part covered by electrodes $\left(\partial \Omega_{\mathrm{e}}\right)$ which generate the electric field.

Field-induced actions can be derived by computing the variation of the electrostatic energy $\mathcal{E}_{\text {el }}$ resulting from a virtual variation of configuration $[46,34]$, i.e.,

$\delta \mathcal{E}_{\mathrm{el}}=\int_{\Omega_{1} \cup \Omega_{2}}-\boldsymbol{f} \cdot \delta \boldsymbol{s} \mathrm{d} V+\int_{\Gamma}-\boldsymbol{f}_{\Gamma} \cdot \delta \boldsymbol{s} \mathrm{d} A$,

where $\boldsymbol{f}$ and $\boldsymbol{f}_{\Gamma}$ denote volume and surface forces, respectively, and $\delta \boldsymbol{s}$ is a virtual displacement compactly supported in $\Omega_{1} \cup \Omega_{2}$. The variation $\delta \mathcal{E}_{\mathrm{el}}$ is performed keeping constant the potential on the electrodes. Accordingly, the following energy functional is considered

$\mathcal{E}_{\mathrm{el}}=\int_{\Omega_{1} \cup \Omega_{2}} \frac{1}{2} \varepsilon \boldsymbol{e} \cdot \boldsymbol{e} \mathrm{d} V+\int_{\Gamma} \frac{1}{2} C \llbracket \psi \rrbracket^{2} \mathrm{~d} A-\sum_{i} Q_{i} \psi_{i}$,

where $\boldsymbol{e}$ is the electric field, $\psi$ is the electric potential, and $Q_{i}$ and $\psi_{i}$ denote the charge and the electric potential on the $i$-th electrode, respectively. Moreover, $\varepsilon=\varepsilon_{k} \varepsilon_{\mathrm{v}}$ in $\Omega_{k}, k=1,2$, the symbol $\cdot$ denotes the scalar product, and $\llbracket \cdot \rrbracket$ denotes the jump of the enclosed quantity across the membrane. The quasi-static approximation, $\boldsymbol{e}=-\nabla \psi$, is assumed, where $\nabla$ denotes the gradient operator. By resorting to the notion of configurational derivative $[33,53]$, it can be shown that

$\delta \mathcal{E}_{\mathrm{el}}=\int_{\Omega_{1} \cup \Omega_{2}} \boldsymbol{T}: \hat{\nabla} \delta \boldsymbol{s} \mathrm{d} V+\int_{\Gamma} t_{\Gamma}\left(\operatorname{div}_{\tau} \delta \boldsymbol{s}\right) \mathrm{d} A$.

Here $\hat{\nabla}$ and $\operatorname{div}_{\tau}$ denote the symmetric gradient and the tangential divergence operators, respectively, $\boldsymbol{T}$ is the Maxwell stress tensor

$\boldsymbol{T}=\varepsilon\left[\boldsymbol{e} \otimes \boldsymbol{e}-\frac{1}{2}|\boldsymbol{e}|^{2} \boldsymbol{I}\right]$,

where $|\cdot|$ denotes modulus and $\boldsymbol{I}$ is the identity tensor, and $t_{\Gamma}$ is given by

$t_{\Gamma}=-\frac{1}{2} C \llbracket \psi \rrbracket^{2}$.

The latter term, being dual to local area variations, can be regarded as a hydrostatic membrane stress, and does not induce any deformation due to the local area conservation hypothesis (see Section 3.2.1).

Upon integration by parts, the first term in (3) can be rewritten as

$$
\begin{aligned}
\int_{\Omega_{1} \cup \Omega_{2}} \boldsymbol{T}: \hat{\nabla} \delta s \mathrm{~d} V= \\
\quad \int_{\Omega_{1} \cup \Omega_{2}}-\operatorname{div} \boldsymbol{T} \cdot \delta \boldsymbol{s} \mathrm{d} V+\int_{\Gamma}-\llbracket \boldsymbol{T n} \rrbracket \cdot \delta \boldsymbol{s} \mathrm{d} A,
\end{aligned}
$$

yielding the volume forces $\boldsymbol{f}$ and the surface forces $\boldsymbol{f}_{\Gamma}$ induced by the electric field as

$$
\boldsymbol{f}=\operatorname{div} \boldsymbol{T}, \quad \boldsymbol{f}_{\Gamma}=\llbracket \boldsymbol{T n} \rrbracket,
$$

where div denotes the divergence operator and $\boldsymbol{n}$ is the normal unit vector to $\Gamma$ pointing into $\Omega_{2}$. It is a simple matter to show that $[46]$

$f=-\frac{1}{2}(\nabla \varepsilon) e \cdot e$.

As a consequence, under the assumption of constant permittivities, volume forces vanish and only the surface forces $\boldsymbol{f}_{\Gamma}$ acting on the cell membrane have to be considered as load in the mechanical module (Section 3). From $(7)_{2}$ and (4), their computation requires the knowledge of the electric field distribution (see Section 2.2). Moreover, in case of time-harmonic electric field

$\boldsymbol{e}=\boldsymbol{A} \cos (\omega t+\varphi)$,

the time-averaged counterpart of the Maxwell stress tensor has to be adopted $[51,49]$, given by

$<\boldsymbol{T}>=\frac{1}{4} \varepsilon\left[(\boldsymbol{E} \otimes \overline{\boldsymbol{E}}+\overline{\boldsymbol{E}} \otimes \boldsymbol{E})-|\boldsymbol{E}|^{2} \boldsymbol{I}\right]$,

where $\boldsymbol{E}=\boldsymbol{A} \mathrm{e}^{\mathrm{i} \varphi}$ is the electric field phasor and the overline denotes complex conjugation. 


\subsection{Electric field distribution}

In the Fourier domain, the electrical problem is stated as follows:

$$
\begin{aligned}
& -\operatorname{div}\left(\sigma^{*} \nabla \Psi\right)=0, \quad \text { in } \Omega_{1} \cup \Omega_{2} ; \\
& \llbracket \sigma^{*} \nabla \Psi \cdot \boldsymbol{n} \rrbracket=0, \quad \text { on } \Gamma \text {; } \\
& Y \llbracket \Psi \rrbracket=\sigma^{*} \nabla \Psi \cdot \boldsymbol{n}, \quad \text { on } \Gamma,
\end{aligned}
$$

where $\Psi$ is the electric potential phasor and $\sigma^{*}=\sigma_{k}^{*}$ in $\Omega_{k}, k=1,2$. Equation (11) governs the electric conduction in the cell cytoplasm and in the fluid; (12) accounts for the continuity of the current flux density through the cell membrane; (13) describes the membrane electric behavior.

An insulating boundary condition is applied on the boundaries not covered by electrodes

$\sigma^{*} \nabla \Psi \cdot \boldsymbol{n}=0, \quad$ on $\partial \Omega_{\mathrm{ne}}$.

On the $i$-th electrode $\left(\partial \Omega_{\mathrm{e}_{i}}\right)$, the following electrode equation holds [45]

$Y_{\mathrm{e}}\left(\Psi_{i}-\Psi\right)=\sigma^{*} \nabla \Psi \cdot \boldsymbol{n}, \quad$ on $\partial \Omega_{\mathrm{e}_{i}}$,

where $Y_{\mathrm{e}}=G_{\mathrm{e}}+\mathrm{i} \omega C_{\mathrm{e}}$ is the electrode double-layer admittance per unit area, expressed in terms of the conductance $G_{\mathrm{e}}$ and capacitance $C_{\mathrm{e}}$ per unit area; $\Psi_{i}$ is the prescribed electrode potential; the right-hand side is the current density through the electrode. In the radiofrequency range, $G_{\mathrm{e}}$ is usually negligible with respect to $\omega C_{\mathrm{e}}$.

Problem (11)-(15) is recast into the following weak formulation

$$
\begin{aligned}
& \int_{\Omega_{1} \cup \Omega_{2}} \sigma^{*} \nabla \Psi \cdot \nabla \Phi \mathrm{d} V+\int_{\Gamma} Y \llbracket \Psi \rrbracket \llbracket \Phi \rrbracket \mathrm{d} A \\
& \quad+\sum_{i} \int_{\partial \Omega_{\mathrm{e}_{i}}} Y_{\mathrm{e}} \Psi \Phi \mathrm{d} A=\sum_{i} \int_{\partial \Omega_{\mathrm{e}_{i}}} Y_{\mathrm{e}} \Psi_{i} \Phi \mathrm{d} A
\end{aligned}
$$

where $\Phi$ is an arbitrary test function. Exploiting this formulation, the potential distribution $\Psi$ induced by prescribed electrode potentials $\Psi_{i}$ can be uniquely determined [45], whence the electric field follows

$\boldsymbol{E}=-\nabla \Psi$.

As detailed in Section 4.1, particular care is taken in the implementation of the latter relationship.

\section{Mechanical module}

\subsection{Mechanical problem}

The structure of a RBC can be characterized as a liquid capsule enclosed by a biologic membrane. This membrane is composed of a phospholipid bilayer and an underlying spectrin network, the cytoskeleton, coupled to each other by integral membrane proteins (Figure 2(a)). (a)

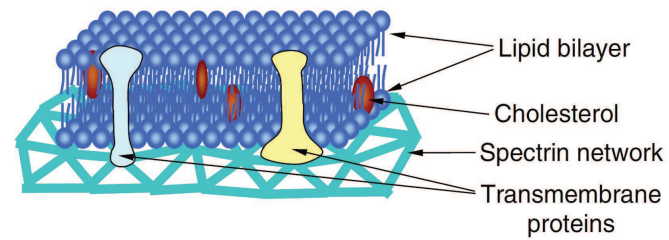

(b)

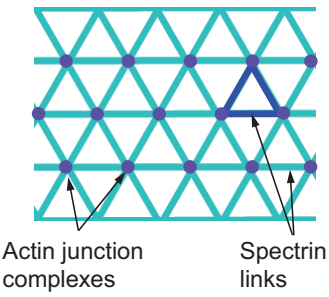

(c)

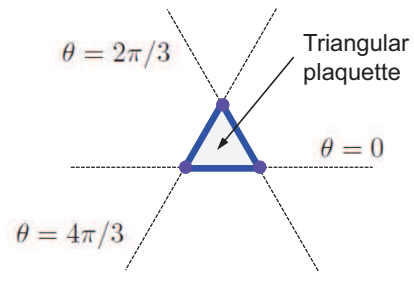

Fig. 2 (a) Schematic drawing of the RBC membrane structure (not to scale), reprinted with permission from [14]. (b) Idealised regular triangular network. (c) Representative triangle

From a mechanical point of view, the spectrin network accounts for the in-plane shear stiffness of the membrane. On the other hand, the lipid bilayer resembles a fluid-like membrane, and is responsible of membrane bending stiffness and total surface area conservation [37]. Moreover, assuming that the connection between spectrin network and lipid bilayer is dense enough, the stricter constraint of local surface area conservation can be enforced [24]. Finally, the nearly incompressible cytosol inside the RBC determines the total volume conservation constraint [18].

Such arguments motivate the definition of the following energy potential, which governs the quasi-static mechanical evolution of the RBC $[18,37,24]$ :

$\mathcal{E}=W_{\text {in-plane }}+W_{\text {bend }}+W_{\text {vol }}+\mathcal{E}_{\text {ext }}$,

where $W_{\text {in-plane }}$ and $W_{\text {bend }}$ denote the membrane inplane and bending energies, respectively, $W_{\text {vol }}$ is a volume penalty energy term imposing the total volume conservation constraint and $\mathcal{E}_{\text {ext }}$ is the potential of external loads. The local surface area conservation constraint is included in the potential (18) as a contribution to the in-plane energy $W_{\text {in-plane }}$.

Introducing the in-plane energy density $w_{\text {in-plane }}$ per unit reference area $[17,5,18,35]$ and the bending energy density $w_{\text {bend }}$ per unit current area [31], it follows that:

$W_{\text {in-plane }}=\int_{A_{0}} w_{\text {in-plane }} \mathrm{d} A, \quad W_{\text {bend }}=\int_{A} w_{\text {bend }} \mathrm{d} A$,

where $A_{0}$ [resp. $\left.A\right]$ is the surface area in the reference [resp. current] configuration. Expressions of $w_{\text {in-plane }}$ 
and $w_{\text {bend }}$ are discussed in Section 3.2. The volume penalty energy term is defined as [18]:

$W_{\mathrm{vol}}=\frac{1}{2} k_{\mathrm{vol}} \frac{\left(V-V_{0}\right)^{2}}{V_{0}}$,

where $V_{0}[$ resp. $V]$ is the total cell volume in the reference [resp. current] configuration and $k_{\mathrm{vol}}$ is a suitable penalty parameter.

\subsection{Constitutive behaviour}

Following the formulation presented in Section 3.1, the definition of the RBC membrane constitutive law consists in the selection of in-plane and bending energy densities. In particular, starting from the so-called spectrin network model $[17,5,18,35]$, the in-plane energy density $w_{\text {in-plane }}$ is obtained by means of homogenization of the Helmholtz free energy of the microstructure, as detailed in Section 3.2.1. Moreover, the bending energy density $w_{\text {bend }}$ is assumed to obey the Helfrich model [31], as described in Section 3.2.2.

\subsubsection{In-plane behavior}

The in-plane behavior of the RBC membrane is determined by the spectrin network and the local surface area conservation constraint imposed by the lipid bilayer. According to the spectrin network model [17, $5,18,35]$, the former is modeled as an idealised regular triangular network, comprising an assembly of actin junction complexes connected to each other by spectrin links (Figure 2(b)). The model is completed with the assumption that the triangular plaquettes bounded by a triplet of mutually connected actin junction complexes are attached to the lipid bilayer (Figure 2(c)). Consequently, the equilibrium condition of the actin junction complexes under the attractive forces exerted by the spectrin links and the repulsive ones arising from the lipid triangular plaquettes, determines the surface area of the network in its natural state. Modifications of such surface area are locally prevented by the lipid bilayer.

On the basis of the Helmholtz free energy stored in each constituent, a microstructurally-based homogenized constitutive law, expressing the relationship between macroscopic stress and strain measures, is derived here for such composite medium. To this end, the contributions to the Helmholtz free energy arising from each constituent are investigated:

$i$. the total entropic free energy stored in the spectrin proteins is estimated by means of the worm-like chain model $[41,6]$. Accordingly, the contribution from a single spectrin link is:

$\mathcal{W}_{\mathrm{WLC}}=\frac{k_{\mathrm{B}} T L_{\max }}{4 p} \frac{3 x^{2}-2 x^{3}}{1-x}, x=L / L_{\max }$,

where $x$ is the current dimensionless chain length, i.e. the current length $L$ normalized by the contour length $L_{\max }$ of the chain, $p$ is the persistency length, $k_{\mathrm{B}}$ is the Boltzmann constant and $T$ is the absolute temperature. Introducing the chain stretch:

$\lambda=L / L_{0}$,

where $L_{0}$ denotes the chain length in the undeformed (stress-free) lattice, the dimensionless length $x$ can be recast as:

$x=\lambda x_{0}, \quad x_{0}=L_{0} / L_{\max }$,

in which $x_{0}$ represents the dimensionless chain length in the undeformed lattice. As a consequence of equation (23), the worm-like chain free energy $\mathcal{W}_{\mathrm{WLC}}$ results to be a function of the stretch $\lambda$ undergone by the spectrin link under consideration:

$\mathcal{W}_{\mathrm{WLC}}=\frac{k_{\mathrm{B}} T L_{0}^{2}}{4 p L_{\max }} \frac{\left(3 L_{\max }-2 \lambda L_{0}\right) \lambda^{2}}{L_{\max }-\lambda L_{0}} ;$

ii. the contribution from a single triangular plaquette to the hydrostatic elastic energy stored in the lipid membrane, is evaluated as [18]:

$\mathcal{W}_{\text {hydr }}=\frac{C_{\mathrm{q}}}{a^{q}}$,

where $a$ is the current area of the plaquette, $q$ is a suitable exponent and $C_{\mathrm{q}}$ is a constant to be determined by prescribing the undeformed configuration of the lattice. Introducing the areal stretch:

$\alpha=a / a_{0}$,

where $a_{0}$ denotes the area of the plaquette in the undeformed lattice, the hydrostatic elastic energy can be recast as:

$\mathcal{W}_{\text {hydr }}=\frac{C_{\mathrm{q}}}{\left(\alpha a_{0}\right)^{q}}$.

It is noted that, because the hydrostatic elastic energy $\mathcal{W}_{\text {hydr }}$ depends only on the areal stretch $\alpha$, there is no contribution of the lipid membrane to the RBC shear stiffness; 
iii. the local surface area conservation constraint imposed by the lipid bilayer is accounted for by considering an additional contribution to the Helmholtz free energy of the RBC membrane, namely [18]:

$\mathcal{W}_{\text {surf }}=\frac{1}{2} k_{\text {surf }} \frac{\left(a-a_{0}\right)^{2}}{a_{0}}$

where $k_{\text {surf }}$ is a penalty parameter. In terms of the areal stretch $\alpha$, the surface energy $\mathcal{W}_{\text {surf }}$ is conveniently recast as:

$$
\mathcal{W}_{\text {surf }}=\frac{1}{2} k_{\text {surf }} a_{0}(\alpha-1)^{2} \text {. }
$$

In order to determine the homogenized energy of the RBC membrane, the triangular spectrin network depicted in Figure 2(b) is considered. Accordingly, a single equilateral triangle can be assumed as representative element. The triangle is assumed to undergo an inplane strain described in terms of the Green-Lagrange strain tensor $\mathbb{E}$. Such strain measure has to be interpreted as a macroscopic variable representative of the average microstructural deformation. Because no strain fluctuation due to local inhomogeneities arises in a regular lattice, the microstructural stretch $\lambda$ along a given direction $\boldsymbol{e}$ and areal stretch $\alpha$ are given by:

$\lambda=\sqrt{1+2 \mathbb{E} e \cdot \boldsymbol{e}}, \quad \alpha=\sqrt{\operatorname{det}(\boldsymbol{I}+2 \mathbb{E})}$.

Assuming that the triangle has the three sides (spectrin links) lying along the directions defined by the polar angles $\theta=\{0,2 \pi / 3,4 \pi / 3\}$, as shown in Figure 2(c), the resulting homogenized free energy density turns out to be:

$w_{\text {in-plane }}=\frac{1}{a_{0}}\left[\sum_{\theta} \frac{1}{2} \mathcal{W}_{\mathrm{WLC}}(\theta)+\mathcal{W}_{\text {hydr }}+\mathcal{W}_{\text {surf }}\right]$.

Here the summation is extended to the three spectrin links, each belonging to two adjacent triangles, and equations (24), (27), (29) and (30) have to be used. Consequently, the macroscopic second Piola-Kirchhoff stress tensor $\mathbb{S}$ results in:

$\mathbb{S}=\nabla_{\mathbb{E}} w_{\text {in-plane }}$

Finally, the constant $C_{\mathrm{q}}$, involved in the computation of the hydrostatic elastic energy (27) stored in the lipid membrane, is determined by imposing that the RBC membrane is stress-free for a vanishing applied strain tensor $\mathbb{E}$. Exploiting equation (32), it is a simple matter to check that [14]:

$C_{\mathrm{q}}=\frac{3}{16} \frac{k_{\mathrm{B}} T a_{0}^{q} L_{\max }}{p q} \frac{x_{0}^{2}\left(6-9 x_{0}+4 x_{0}^{2}\right)}{\left(1-x_{0}\right)^{2}}$.

\subsubsection{Bending behavior}

The bending behaviour of the RBC membrane, completely attributable to the lipid bilayer, is described by means of the Helfrich energy [31]:

$w_{\text {bend }}=\frac{k_{\mathrm{c}}}{2}\left(J-J_{0}\right)^{2}+k_{\mathrm{g}} K$,

where $J$ and $K$ respectively denote total and Gaussian curvature in the current configuration, $J_{0}$ is the reference total curvature, and $k_{\mathrm{c}}, k_{\mathrm{g}}$ are bending elastic moduli. It is recalled that, in terms of the principal curvatures $\left\{k_{1}, k_{2}\right\}$ of the surface membrane, total and Gaussian curvatures result to be $J=k_{1}+k_{2}$ and $K=k_{1} k_{2}$, respectively (e.g., see [19]).

\section{Numerical procedure}

In this section, the numerical procedure adopted in the electrical and mechanical modules as well as their coupling strategy are described.

\subsection{Electrical module}

Formulation (16) is implemented into the commercial finite element code COMSOL Multyphysics. A triangular mesh of the cell membrane $\Gamma$ is generated and used as boundary mesh in the creation of tetrahedral meshes of the intracellular and fluid domains (respectively, $\Omega_{1}$ and $\Omega_{2}$ ). Treating the cell membrane as a two-dimensional interface avoids the need of extra-fine tetrahedral meshes as required in a three-dimensional description of a thin region $[2,9,10]$. Weak Form PDE Physics is adopted, and quadratic Lagrangian elements are used to interpolate the electric potential $\Psi$.

The electric field $\boldsymbol{E}$ on both sides of the membrane $\Gamma$ is computed by the numerical approximation of $\Psi$. In particular, introducing the projector $\boldsymbol{P}_{\tau}=\boldsymbol{I}-\boldsymbol{n} \otimes \boldsymbol{n}$ on the membrane tangent plane, $\boldsymbol{E}$ is decomposed as

$\boldsymbol{E}=\boldsymbol{E}_{\tau}+E_{\boldsymbol{n}} \boldsymbol{n}$,

where $\boldsymbol{E}_{\tau}=\boldsymbol{P}_{\tau} \boldsymbol{E}$ is the tangential field and $E_{\boldsymbol{n}}$ denotes the normal component. The former is computed by means of the COMSOL operator dtang, implementing the tangential derivative of the vector field $\Psi$; the latter is calculated by means of a wise exploitation of (13), i.e.,

$E_{n}=-\frac{Y \llbracket \Psi \rrbracket}{\sigma^{*}}$.

It is worth mentioning that the direct computation $E_{\boldsymbol{n}}=$ $-\nabla \Psi \cdot \boldsymbol{n}$, involving the gradient of the interpolated 
quantity, would yield significantly less accurate results. The field-induced forces acting on the cell membrane, that represent the input to the mechanical module, are computed from $\boldsymbol{E}$ via (10) and $(7)_{2}$.

\subsection{Mechanical module}

\subsubsection{Finite element formulation}

A finite element formulation of the mechanical problem introduced in Section 3.1 is here discussed. To this end, given a triangulation $\bigcup_{e=1}^{N^{e}} A_{0}^{e}$ of the RBC surface in the reference configuration $A_{0}$, the unknown displacement field $\boldsymbol{u}$ at a typical point $p$ is approximated by:

$\boldsymbol{u}(p) \approx \sum_{e=1}^{N^{e}} \chi^{e} \boldsymbol{u}^{e}\left(\boldsymbol{a}^{e}, p\right)$

In equation (37), $\boldsymbol{u}^{e}$ denotes the interpolation of the displacement field over the element $A_{0}^{e}$, depending on element nodal displacements $\boldsymbol{a}^{e}$ and point $p$, and $\chi^{e}$ is the characteristic function associated to the same element. Using such representation formula for the displacement field $\boldsymbol{u}$, it is straightforward to derive:

$$
\begin{aligned}
\mathbb{E} & =\mathbb{E}(\boldsymbol{a}, p), \\
J & =J(\boldsymbol{a}, p), \quad K=K(\boldsymbol{a}, p), \\
V & =V(\boldsymbol{a}),
\end{aligned}
$$

that is, a consistent approximation of Green-Lagrange strain tensor, of total and Gaussian curvatures, and of cell volume, in terms of nodal displacements $\boldsymbol{a}$. Accordingly, the potential energy (18) can be computed by:

$\mathcal{E}=\sum_{e=1}^{N^{e}}\left\{W_{\text {in-plane }}^{e}+W_{\text {bend }}^{e}+\mathcal{E}_{\text {ext }}^{e}\right\}+W_{\text {vol }}$,

where $(\cdot)^{e}$ denotes the element counterpart of the relevant quantity.

Imposing the stationary condition of the discretized potential energy (39), it follows that the nodal displacements $\boldsymbol{a}$ satisfy the equilibrium equation:

$\mathbf{0}=\underset{e=1}{\mathcal{N}^{e}}\left\{\boldsymbol{q}_{\mathrm{ext}}^{e}-\boldsymbol{q}_{\mathrm{int}}^{e}\right\}-\boldsymbol{q}_{\mathrm{vol}}$,

in which $\mathcal{A}$ denotes the standard assembly operator of the finite element method, $\boldsymbol{q}_{\text {int }}^{e}\left[\right.$ resp. $\left.\boldsymbol{q}_{\text {ext }}^{e}\right]$ are the nodal internal [resp. external] forces work-conjugated to element nodal displacements $\boldsymbol{a}^{e}$, and $\boldsymbol{q}_{\mathrm{vol}}$ are the nodal reactions due to volume constraint, work-conjugated to the nodal displacements $\boldsymbol{a}$. In particular, the element nodal internal forces $\boldsymbol{q}_{\text {int }}^{e}$ are defined by:

$$
\begin{aligned}
\boldsymbol{q}_{\text {int }}^{e} \cdot \delta \boldsymbol{a}^{e} & =\delta W_{\text {in-plane }}^{e}+\delta W_{\text {bend }}^{e} \\
& =\int_{A_{0}^{e}} \delta w_{\text {in-plane }} \mathrm{d} A \\
& +\int_{A_{0}^{e}}\left\{\delta w_{\text {bend }} \alpha+w_{\text {bend }} \delta \alpha\right\} \mathrm{d} A,
\end{aligned}
$$

where $\alpha$ is the areal stretch defined in equation $(30)_{2}$, the element nodal external forces $\boldsymbol{q}_{\text {ext }}^{e}$ satisfy:

$-\boldsymbol{q}_{\mathrm{ext}}^{e} \cdot \delta \boldsymbol{a}^{e}=\delta \mathcal{E}_{\mathrm{ext}}^{e}$,

and the nodal reactions due to volume constraint $\boldsymbol{q}_{\mathrm{vol}}$ follow from:

$\boldsymbol{q}_{\mathrm{vol}} \cdot \delta \boldsymbol{a}=\delta W_{\mathrm{vol}}=k_{\mathrm{vol}} \frac{V-V_{0}}{V_{0}} \delta V$.

The derivation of structural consistent stiffness matrix, needed e.g. by Newton's method of solution of global equilibrium equations, requires the linearization of equations (41)-(43) with respect to the nodal displacements $\boldsymbol{a}$. With regard to the element nodal internal forces $\boldsymbol{q}_{\mathrm{int}}^{e}$, it turns out that:

$$
\begin{aligned}
\boldsymbol{K}_{\boldsymbol{q}_{\text {int }}^{e}} \Delta \boldsymbol{a}^{e} & \cdot \delta \boldsymbol{a}^{e}:=\Delta \boldsymbol{q}_{\text {int }}^{e} \cdot \delta \boldsymbol{a}^{e} \\
& =\int_{A_{0}^{e}} \Delta \delta w_{\text {in-plane }} \mathrm{d} A \\
& +\int_{A_{0}^{e}}\left\{\Delta \delta w_{\text {bend }} \alpha+\delta w_{\text {bend }} \Delta \alpha\right\} \mathrm{d} A \\
& +\int_{A_{0}^{e}}\left\{\Delta w_{\text {bend }} \delta \alpha+w_{\text {bend }} \Delta \delta \alpha\right\} \mathrm{d} A
\end{aligned}
$$

where $\Delta$ denotes the linearization operator. Analogously, the linearization of the element nodal external forces $\boldsymbol{q}_{\text {ext }}^{e}$ yields:

$\boldsymbol{K}_{\boldsymbol{q}_{\mathrm{ext}}^{e}} \Delta \boldsymbol{a}^{e} \cdot \delta \boldsymbol{a}^{e}:=\Delta \boldsymbol{q}_{\mathrm{ext}}^{e} \cdot \delta \boldsymbol{a}^{e}$.

However, in the present work, the coupling between electrical and mechanical modules is treated through the fixed-point iteration scheme discussed in Section 4.3, and such stiffness contribution need not be accounted for. On the other hand, its computation would be necessary for a monolithic solution strategy of the coupled problem adopting Newton's method. Furthermore, the linearization of the nodal reactions due to volume constraint $\boldsymbol{q}_{\mathrm{vol}}$ gives:

$$
\begin{aligned}
\boldsymbol{K}_{\boldsymbol{q}_{\mathrm{vol}}} \Delta \boldsymbol{a} \cdot \delta \boldsymbol{a} & :=\Delta \boldsymbol{q}_{\mathrm{vol}} \cdot \delta \boldsymbol{a} \\
& =k_{\mathrm{vol}} \frac{\Delta V}{V_{0}} \delta V+\frac{V-V_{0}}{V_{0}} \Delta \delta V .
\end{aligned}
$$

Finally, a standard finite element assemblage of stiffness matrices reported in equations (44)-(46), yields the desired structural consistent stiffness matrix. 


\subsubsection{Element formulation}

According to equations (37)-(38), the computation of a consistent approximation of Green-Lagrange strain tensor, and of total and Gaussian curvatures, requires a suitable interpolation of the displacement field at the element level.

The element formulation turns out to be greatly simplified when developed within a corotational framework, able to handle large element rigid-body motions. In particular, the corotational procedure can be visualized like a two-way filter that $i$ ) removes the rigid-body contribution from the element nodal displacements before the finite element kernels use them, and $i i$ ) brings back to the overall level the quantities returned by the element routine [25]. A brief description of the corotational framework is given in Appendix A.

With a slight abuse of notation and dropping out the element index $e$, the element displacement field and element nodal displacements resulting after filtering out the rigid-body contribution, are still denoted by $\boldsymbol{u}$ and $\boldsymbol{a}$, respectively. In particular, the vector $\boldsymbol{a}$ can be arranged as:

$\boldsymbol{a}=\left\{\boldsymbol{u}_{1} ; \boldsymbol{u}_{2} ; \boldsymbol{u}_{3}\right\}$

where $\boldsymbol{u}_{i}, i=1,2,3$, is the displacement vector of the typical node $V_{i}$ in the reference configuration, and the semicolon symbol denotes column stacking. As a consequence, the transformation to be handled at the finite element level maps the reference element nodes $V_{i}$ into the current points $\bar{V}_{i}=V_{i}+\boldsymbol{u}_{i}$.

For convenience, an orthonormal frame with origin $O$ at the element centroid, and axes:

$\boldsymbol{g}_{1}=\frac{V_{2}-V_{1}}{\left\|V_{2}-V_{1}\right\|}, \boldsymbol{g}_{2}=\boldsymbol{g}_{3} \times \boldsymbol{g}_{1}, \boldsymbol{g}_{3}=\frac{\boldsymbol{g}_{1} \times\left(V_{3}-V_{1}\right)}{\left\|\boldsymbol{g}_{1} \times\left(V_{3}-V_{1}\right)\right\|}$,

is introduced. Furthermore, the position vector in the reference configuration is denoted by $\boldsymbol{X}=(X, Y, Z)$.

The rigid-body motion filtered out by the corotational filter is here chosen such that the reference triangle $V_{1} V_{2} V_{3}$ and the deformed triangle $\bar{V}_{1} \bar{V}_{2} \bar{V}_{3}$ are made coplanar and share their centroid (see Appendix A). Accordingly, it turns out that:

$z_{1}=z_{2}=z_{3}=0, \quad \sum_{i=1}^{3} x_{i}=\sum_{i=1}^{3} y_{i}=0$,

where $\boldsymbol{x}=(x, y, z)$ denotes the position vector in the deformed configuration.

The in-plane components of the displacement field $\boldsymbol{u}$,

$u(X, Y)=\boldsymbol{u}(\boldsymbol{X}) \cdot \boldsymbol{g}_{1}, \quad v(X, Y)=\boldsymbol{u}(\boldsymbol{X}) \cdot \boldsymbol{g}_{2}$,

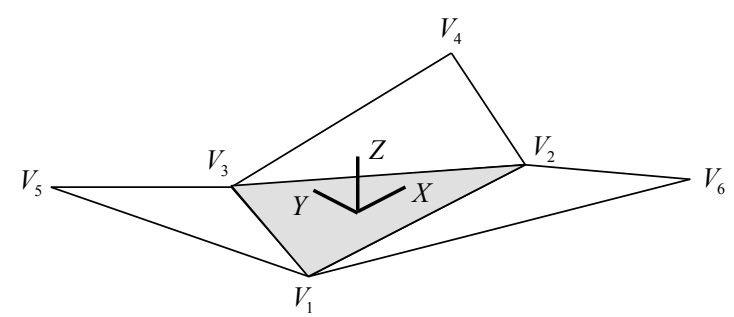

Fig. 3 Patch associated to element $V_{1} V_{2} V_{3}$ in the reference configuration

are approximated with the constant strain triangle (CST) interpolation [48] of the nodal in-plane displacements:

$\boldsymbol{u}_{\mathrm{m}}=\left\{u_{1} ; v_{1} ; u_{2} ; v_{2} ; u_{3} ; v_{3}\right\}$,

in which:

$u_{i}=\boldsymbol{u}_{i} \cdot \boldsymbol{g}_{1}, \quad v_{i}=\boldsymbol{u}_{i} \cdot \boldsymbol{g}_{2}, \quad i=1,2,3$.

Hence, the Green-Lagrange strain tensor $\mathbb{E}$ can be derived by:

$\mathbb{E}=\frac{1}{2}\left(\mathbb{F}^{\mathrm{T}} \mathbb{F}-\boldsymbol{I}\right), \quad \mathbb{F}=\boldsymbol{I}+\mathbb{H}, \quad \mathbb{H}=\nabla_{\boldsymbol{X}}(u, v)$,

where $\mathbb{F}$ and $\mathbb{H}$ are deformation and displacement gradient, respectively.

In order to estimate the curvature tensor $\boldsymbol{\chi}$, and consequently the total curvature $J$ and the Gaussian curvature $K$, an original rotation-free formulation is here proposed. Differently from the standard finite element format, rotation-free formulations are characterized by a distinction between integration and interpolation domains (e.g., see $[43,55])$. In particular, the integration domain is the element under investigation, namely the triangle $V_{1} V_{2} V_{3}$, whereas the interpolation domain is assumed to be the patch also comprising the three adjacent triangles $V_{1} V_{6} V_{2}, V_{2} V_{4} V_{3}$ and $V_{3} V_{5} V_{1}$ (see Figure 3). Accordingly, the interpolation parameters coincide with the patch nodal displacements:

$\boldsymbol{u}_{\mathrm{b}}=\left\{\boldsymbol{u}_{1} ; \ldots ; \boldsymbol{u}_{6}\right\}$

Relying on conditions (49), the deformed patch can be effectively approximated by means of the paraboloid:

$z=c_{1}+c_{2} x+c_{3} y+c_{4} x^{2}+c_{5} y^{2}+c_{6} x y$,

the constants $c_{i}, i=1, \ldots, 6$, being determined by the following interpolation conditions:

$z\left(x_{i}, y_{i}\right)=z_{i}=\left(\bar{V}_{i}-O\right) \cdot \boldsymbol{g}_{3}, \quad i=1, \ldots, 6$.

The curvature tensor $\chi$ over the deformed triangle $\bar{V}_{1} \bar{V}_{2} \bar{V}_{3}$ is then estimated by the curvature of the paraboloid at the origin. Provided that coefficients $c_{2}$ and $c_{3}$ are 


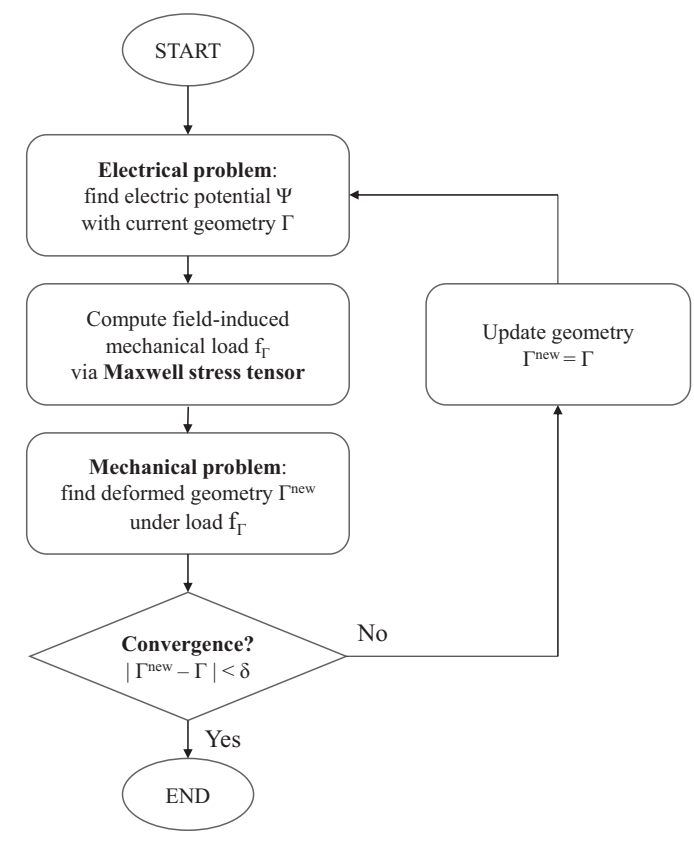

Fig. 4 Flow chart of electro-mechanical coupling (see text for explanation)

sufficiently small (condition met in the limit of an increasingly finer mesh), the latter can be approximated with the Hessian of the function (55), whence:

$\chi=\nabla \nabla_{\boldsymbol{x}} z(O), \quad J=\operatorname{tr} \chi, \quad K=\operatorname{det} \chi$

It is worth pointing out that the corotational framework simplifies the differentiation of total and Gaussian curvatures with respect to element nodal displacements, as needed in the element computations, i.e. in equations (41) and (44). In fact, the interpolation (55) remains effective also for a variation of the deformed configuration, being any rotation of the paraboloid axis accounted for (and purged out) by the corotational filter.

\subsection{Electro-mechanical coupling}

The coupling between the electrical and mechanical modules is implemented by means a fixed-point iteration scheme (Figure 4). For given applied electrode potentials $\Psi_{i}$, the electric problem (16) is initially solved on the domain defined by the reference configuration of the cell membrane $\Gamma$. The corresponding field-induced actions $\boldsymbol{f}_{\Gamma}$ are then computed as the jump of the tractions generated by the time-averaged Maxwell stress tensor on the cell membrane (equations $(7)_{2}$ and (4)). The latter surface forces define the potential of the external load $\mathcal{E}_{\text {ext }}$ in the mechanical problem (18), whose solution provides an updated cell-membrane geometry
Table 1 Electric parameters adopted for cell and electrodes [27], and for extracellular fluid [21]

\begin{tabular}{llllll}
\hline$\sigma_{1}(\mathrm{~S} / \mathrm{m})$ & $\varepsilon_{1}$ & $C\left(\mathrm{mF} / \mathrm{m}^{2}\right)$ & $C_{\mathrm{e}}\left(\mathrm{mF} / \mathrm{m}^{2}\right)$ & $\sigma_{2}(\mathrm{~S} / \mathrm{m})$ & $\varepsilon_{2}$ \\
\hline 0.5 & 60 & 10 & 144 & 0.05 & 80 \\
\hline
\end{tabular}

$\Gamma^{\text {new }}$. If the distance between $\Gamma$ and $\Gamma^{\text {new }}$ is lower than a prescribed tolerance $\delta$, the numerical procedure stops, otherwise, a new iteration is performed starting from the updated cell-membrane geometry $\Gamma^{\text {new }}$. In particular, in the convergence check, the norm of the relative difference between the nodal coordinates of $\Gamma$ and $\Gamma^{\text {new }}$ is considered, with a tolerance $\delta=0.001$.

If the solution cannot converge within a reasonable number of iterations, or if intermediate solutions given at uniform intervals of the applied electrode potentials $\Psi_{i}$ are desired, the latter are subdivided into multiple potential-increment steps. At the end of each increment, a converged intermediate solution is obtained, which in turn is used to start the fixed-point iteration for the solution of the next potential increment. This reduces the degree of nonlinearity from an intermediate solution state to another and enhances the chance of obtaining the ultimate solution under the electrode potentials $\Psi_{i}$.

\section{Numerical results}

In this section, numerical results are reported. First, the independent validation of the electrical and mechanical modules is performed. In particular, the problem of a coated ellipsoid in a uniform electric field and the deformation of a RBC by optical tweezers are considered, respectively. Then, an in-silico experiment of erythrocyte electro-deformation is presented, showing the effectiveness and soundness of the proposed approach.

5.1 Electrical module validation: coated ellipsoid in uniform electric field

The problem of an ellipsoidal particle with semidiameters $a>b>c$, coated with a membrane having finite admittance, and embedded in a uniform electric field $\boldsymbol{E}_{\infty}$ established in the infinite space, is considered. Its analytical solution, reported in Appendix B, was used as a benchmark for the numerical results supplied by the electrical module. In particular, an ellipsoid with diameters $2 a=14.1 \mu \mathrm{m}, 2 b=4.64 \mu \mathrm{m}, 2 c=2.44 \mu \mathrm{m}$ was considered. Those values were chosen to mimic the deformed configuration of a RBC under applied electric field (see Section 5.3). The ellipsoid was located at 
(a)

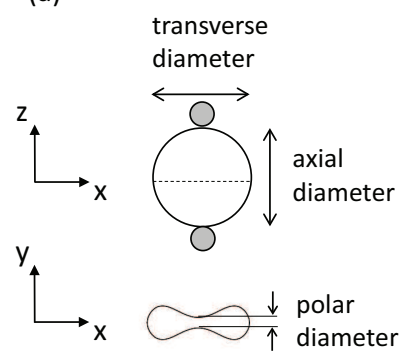

(b)

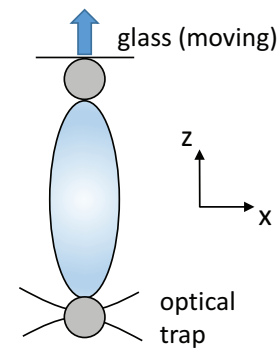

Fig. 5 Schematic representation of optical tweezers experimental set-up. (a) Initial cell geometry and definition of axial, transverse and polar diameters. (b) Stretch test: one bead is optically trapped, while the other one is anchored to the surface of a moving glass slide

Table 2 Parameters of the RBC meridional section [22]

\begin{tabular}{llll}
\hline$R(\mu \mathrm{m})$ & $C_{0}$ & $C_{1}$ & $C_{2}$ \\
\hline 3.91 & 0.207161 & 2.002558 & -1.122762 \\
\hline
\end{tabular}

the center of a parallelepipedic domain whose sides are 80 times the ellipsoid semiaxes. It was subject to an electric field parallel to its major axis, with intensity $E_{\infty}=0.125 \mathrm{~V} / \mu \mathrm{m}$ and frequency $500 \mathrm{kHz}$. The electric field was generated by an applied voltage between the parallelepiped faces orthogonal to the ellipsoid major axis, whereas insulating condition was applied on the other faces. The electric parameters used in the computation are reported in Table 1. Due to symmetry, one-eight of the system was studied.

The relative errors, in the $L^{2}$ norm, of the electric potential $\Psi$ and of the surface force density $\boldsymbol{f}_{\Gamma}$ turned out to be, respectively, $7.1 \times 10^{-3}$ and $6.7 \times 10^{-2}$ for a coarse mesh (7,089 nodal points, 34,494 linear tetrahedral elements, 444 linear triangular elements on the ellipsoid surface), and, respectively, $4.5 \times 10^{-4}$ and $2.1 \times$ $10^{-2}$ for a fine mesh $(510,051$ nodal points, 2,916,394 linear tetrahedral elements, 14,101 linear triangular elements on the ellipsoid surface). These errors can be mainly ascribed to the difference in the boundary condition between the analytical and numerical solutions.

The magnitude of the opposite resultant forces acting upon the two semi-ellipsoids on either side of the plane $z=0$ was computed and turned out to be $231 \mathrm{pN}$.

\subsection{Mechanical module validation: large deformation} of the RBC by optical tweezers

Deformation imposed by optical tweezers provides a useful means for the study of single cell mechanics under a variety of well-controlled stress-states [42]. While

(a)

(b)
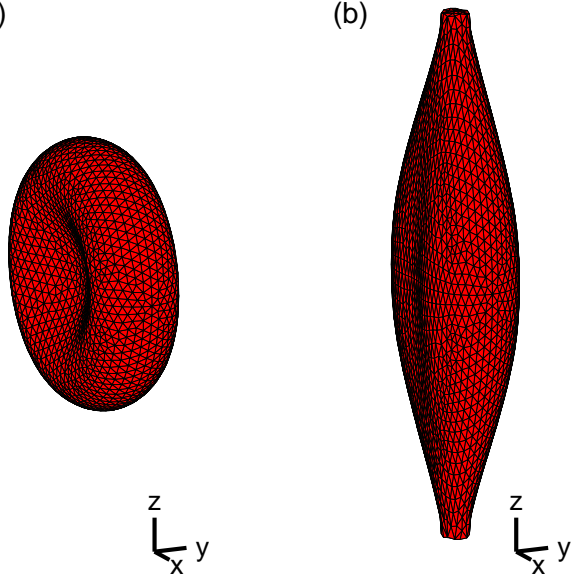

Fig. 6 Numerical simulation of the large deformation of a RBC using optical tweezers. (a) Reference configuration. (b) Deformed configuration under maximum load $\left(F_{\max }=\right.$ $200 \mathrm{pN})$

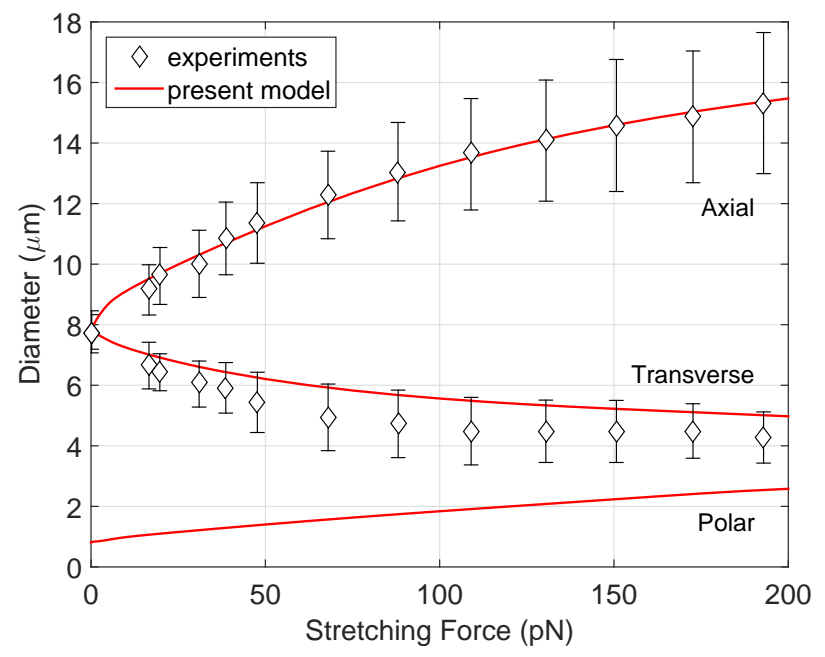

Fig. 7 Diameters versus stretching force during the large deformation of a RBC using optical tweezers. The present numerical results are compared with the experimental data reported in [42] (not including the polar diameter)

early studies involved primarily small elastic deformation at low applied forces, the possibility of inducing large elastic deformation in human RBCs using optical tweezers was demonstrated in $[15,39,38,42]$. In those papers, forces as high as about $193 \pm 20 \mathrm{pN}$ were estimated to result in strains of the order of $100 \%$ in the cell. The experimental procedure adopted to stretch the cell is as follows (Figure 5). Two silica microbeads are non-specifically attached to the RBC at diametrically opposite points. One bead is anchored to the surface of a glass slide, the other one is trapped using a laser beam. While the trapped bead remains stationary, moving the slide with the anchored bead stretches the cell. 
Table 3 Parameters defining the constitutive behaviour of RBC membrane. [24,23]

Here $\beta=\left(k_{\mathrm{B}} T\right)^{-1}, k_{\mathrm{B}}$ is the Boltzmann constant, $a_{0}=L_{0}^{2} \sqrt{3} / 4, V_{0}=75.3 \mu \mathrm{m}^{3}$ (reference RBC volume)

\begin{tabular}{lllllllll}
\hline$L_{\max }(\mathrm{nm})$ & $L_{0}(\mathrm{~nm})$ & $p(\mathrm{~nm})$ & $q$ & $T(\mathrm{~K})$ & $\beta k_{\mathrm{c}}$ & $k_{\mathrm{g}} / k_{\mathrm{c}}$ & $\beta a_{0} k_{\mathrm{surf}}$ & $\beta V_{0} k_{\mathrm{vol}}$ \\
\hline 166.1 & 75.5 & 18.7 & 1 & 296 & 48.9 & $-4 / 3$ & 263.3 & $1.34 \times 10^{8}$ \\
\hline
\end{tabular}

In the in-silico experiment, the biconcave RBC geometric model (Figure 6(a)) was obtained by revolution of the meridional section [22]:

$f(r)=\frac{R}{2} \sqrt{1-\left(\frac{r}{R}\right)^{2}}\left[C_{0}+C_{1}\left(\frac{r}{R}\right)^{2}+C_{2}\left(\frac{r}{R}\right)^{4}\right]$,

whose parameter values are reported in Table 2. The RBC mechanical behaviour was modelled as described in Section 3.2. Parameters adopted in the simulations are reported in Table 3. A detailed discussion of their values along with the experimental fitting procedures leading to their determination can be found in $[18,37$, $24,23]$. The load imposed by each silica bead was simulated as a force $F$ distributed on a circular contact area of diameter $D=1.1 \mu \mathrm{m}$. Equal $z$-displacement was imposed at the interested nodes. Sixteen uniform loadsteps, with internal adaptive sub-stepping, were considered, up to a maximum force level $F_{\max }=200 \mathrm{pN}$. Due to symmetry, one-eight of the cell was studied, with a mesh of 524 elements.

Figure 6(b) shows the deformed configuration of the RBC under maximum load. The behaviour of transverse, axial and polar diameters as functions of the applied force is shown in Figure 7, where the experimental data provided in [42] are also reported for comparison. Excellent agreement is found for the axial diameter, whereas a small discrepancy is noticed between simulated and experimental transverse diameters, with experimental data providing lower values. As suggested in [24], this is probably a consequence of the optical measurements being performed from only a single observation angle, that may result in under-prediction of the maximum transverse diameter in presence of rotation of the RBC during the stretch test. However, the simulation results remain within the experimental error bars. The behaviour of the polar diameter, not reported in the experimental data, highlights a progressive change from the cell biconcave shape to a more rounded shape. Generally, a hardening behaviour with increasing force is noticed, mainly due to the nonlinear constitutive properties of the spectrin network.

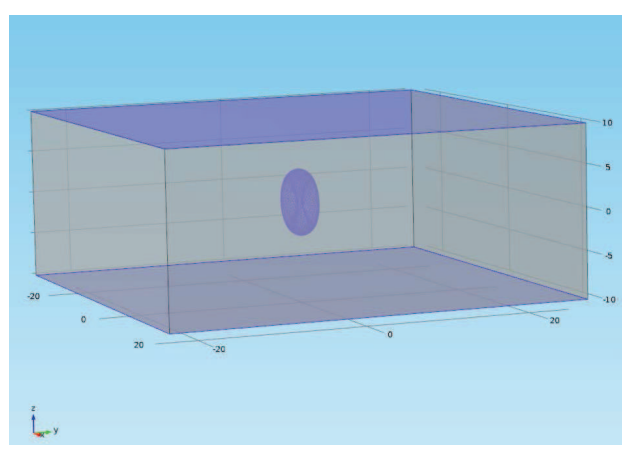

Fig. 8 In silico electro-deformation experiment: a RBC, suspended in a conducting medium, is placed between two facing electrodes, and a voltage is applied between them. (COMSOL geometric model) (a)

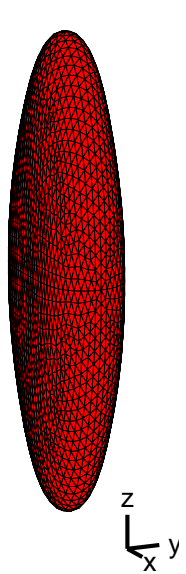

(b)

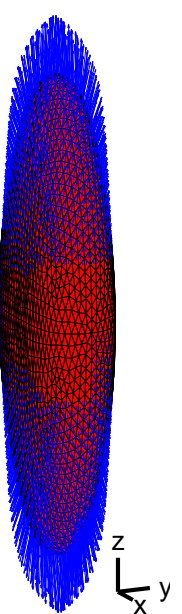

Fig. 9 Electro-deformation of a RBC. (a) Deformed configuration under maximum applied potential $\left(\Psi_{\max }=2.5 \mathrm{~V}\right.$ $@ 500 \mathrm{kHz}$ ). (b) Visualization of field-induced mechanical actions

\subsection{Electro-deformation of a RBC}

A RBC, suspended in a conducting medium, was placed between two $50 \mu \mathrm{m} \times 50 \mu \mathrm{m}$ facing electrodes, that are $20 \mu \mathrm{m}$ apart from each other (Figure 8). A voltage $\Psi$ at $500 \mathrm{kHz}$ was applied between the electrodes. The electric parameters adopted in the computation are reported in Table 1. As typical in electro-deformation experiments, a poorly conductive extracellular fluid was considered [21]. The RBC mechanical behaviour was 


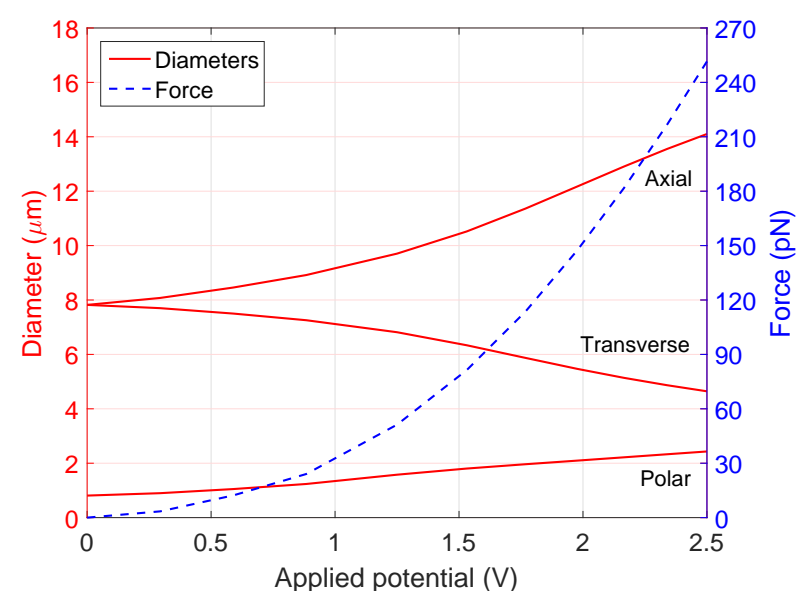

Fig. 10 Diameters and half-cell resultant force versus applied potential during the electro-deformation of a RBC

modelled as in Section 5.2. Due to symmetry, one-eight of the system was studied.

In order to obtain an efficient implementation of the electro-mechanical coupling, the same mesh was adopted to discretize the cell surface in the electrical and mechanical modules. It was composed by 524 triangular elements. Starting from this mesh, a mesh composed by 397,851 quadratic tetrahedral elements was generated to discretize the electric domain. Eight applied-potential steps were considered, with quadratic spacing, up to a maximum value $\Psi_{\max }=2.5 \mathrm{~V}$, corresponding to a field intensity of $0.125 \mathrm{~V} / \mu \mathrm{m}$ in the absence of the cell.

The average number of fixed-point iterations in each load step, needed to reach convergence in the electromechanical coupling scheme, was 3.4.

Figure 9(a) shows the deformed configuration under maximum applied potential, while the mechanical actions induced by the electric field are visualized in Figure 9(b). The magnitude of the resultant force on the top and bottom half cells is shown in Figure 10 as function of the applied voltage $\Psi$. The same figure also reports the axial, transverse, and polar diameters of the deformed RBC. Under the maximum applied voltage, a force magnitude of $252 \mathrm{pN}$ was computed, yielding a deformed RBC configuration with diameters of $14.1 \mu \mathrm{m}, 4.64 \mu \mathrm{m}$, and $2.44 \mu \mathrm{m}$, respectively. Though the electrodes are only $20 \mu \mathrm{m}$ apart from each other, the obtained force magnitude is comparable with the one evaluated in Section 5.1 with reference to an ellipsoid with the same diameters in a uniform electric field at infinity.

Electro-deformation has been recently employed in conjunction with microfluidic systems for biomechanical measurements of RBCs and other cell types [52, $40,11,29,20,21,36]$. An estimate of the applied resul- tant force on half cell is usually computed and related to the experimentally-measured deformation, whence phenomenological indicators of the cell mechanical behaviour are derived. In this context, the modeling and simulation framework here developed represents a valuable tool. In fact, it provides an accurate estimate of the distribution of field-induced actions for cells with arbitrary geometry and without simplifying assumptions on the electric field distribution. This feature can be of aid in the design of optimal electro-deformation systems (e.g. in terms of geometry, medium properties and frequency). Moreover, thanks to the adoption of a microstructurally-based constitutive law, it can be used for the quantitative characterization of microstructural biomechanical properties, by means of an inverse analysis approach.

\section{Conclusions}

In this work, a computational approach for in-silico erythrocyte electro-deformation has been presented. The proposed strategy relies on the Maxwell stress tensor formulation for the accurate computations of the mechanical actions induced by the electric field. In addition, special care is adopted in modeling the cell membrane, that plays a crucial role both from the electrical and the mechanical point of view. In particular, in the electrical module the cell membrane is described as a two-dimensional imperfect interface, by virtue of the capacitive behavior exhibited by the lipid bilayer. This choice avoids the need of extra-fine meshes as required in a three-dimensional description of a thin region, and furthermore allows an efficient computation of the normal component of the electric field. In the mechanical module, a microstructurally-based description of the membrane is adopted, taking into account the costitutive properties of the lipid bilayer and of the underlying spectrin network. As a consequence, the mechanical parameters of the model have a clear physical interpretation. In order to achieve computational efficiency, a homogenization procedure is exploited, under the assumption of regular triangular spectrin network. Finally, a custom rotation-free shell element is adopted, which takes advantage from a corotational formulation for the computation of membrane curvature and its variation.

Several enhancements of the developed tool are envisaged, e.g. accounting for random spectrin network, time-dependent constitutive behaviour, and stiffness contribution arising from configuration-dependent forces. In addition, extension to other cell-lineages is possible, requiring a modification of the constitutive model adopted in the mechanical module. 
As proved by the presented in-silico experiments, the proposed strategy is effective and sound, and constitutes a promising tool towards the development and optimization of microfluidic electro-deformation systems.

\section{A Corotational framework}

The derivation of the corotational approach presented in [7, $8]$ is here briefly reviewed for triangular elements. Let $\boldsymbol{u}_{i}, i=$ $1,2,3$, denote the displacement vector of the typical node $V_{i}$ in the reference configuration. The element nodal parameters are collected into the $9 \times 1$ vector:

$\boldsymbol{a}=\left\{\boldsymbol{u}_{1} ; \boldsymbol{u}_{2} ; \boldsymbol{u}_{3}\right\}$,

where the semicolon symbol denotes column stacking. Moreover, let $\boldsymbol{u}(p)$ be the element displacement field at point $p$ in the reference configuration. In the derivation of corotational finite elements, the deformation $f(p)=p+\boldsymbol{u}(p)$, is multiplicatively decomposed as follows:

$f=r \circ \bar{f}$,

where $r$ is a rigid transformation, characterized by a reference point $G$, a translation vector $\boldsymbol{t}$, and a rotation tensor $\boldsymbol{R}$ :

$r(p)=G+\boldsymbol{t}+\boldsymbol{R}[p-G]$.

Hence, the transformation $\bar{f}$, implying the same deformational motion as $f$, is obtained from the latter after filtering out the rigid motion $r$. Denoting by $\overline{\boldsymbol{u}}(p)=\bar{f}(p)-p$ the filtered displacement field, equations (A.2) and (A.3) yield:

$\boldsymbol{u}=G+\boldsymbol{t}+\boldsymbol{R}[p+\overline{\boldsymbol{u}}-G]-p$.

Then, a suitable interpolation is chosen for $\overline{\boldsymbol{u}}$, characterizing the displacement-based core-element formulation:

$\bar{u}=\bar{u}(\bar{a}, p)$,

where $\overline{\boldsymbol{a}}$ is the filtered counterpart of the element nodal parameters $\boldsymbol{a}$ :

$\overline{\boldsymbol{a}}=\left\{\overline{\boldsymbol{u}}_{1} ; \overline{\boldsymbol{u}}_{2} ; \overline{\boldsymbol{u}}_{3}\right\}$.

Accordingly, the following correspondence rule has to be intended in the notation adopted in Section 4.2:

$u \rightarrow \bar{u}, \quad a \rightarrow \bar{a}$,

amounting at the replacement of element nodal displacements with their filtered counterparts. In particular, the filtered nodal displacements $\overline{\boldsymbol{u}}_{i}$ are obtained by imposing the interpolation conditions $\boldsymbol{u}\left(V_{i}\right)=\boldsymbol{u}_{i}$ and $\overline{\boldsymbol{u}}\left(V_{i}\right)=\overline{\boldsymbol{u}}_{i}$ in (A.4), whence:

$\overline{\boldsymbol{u}}_{i}=\boldsymbol{R}^{\mathrm{T}}\left[V_{i}+\boldsymbol{u}_{i}-(G+\boldsymbol{t})\right]-\left(V_{i}-G\right)$.

The core-element computations, i.e. equation (41), provide the nodal internal-force vector $\overline{\boldsymbol{q}}_{\text {int }}$ work-conjugated to the filtered modal parameters $\overline{\boldsymbol{a}}$. Since the rigid body motion does not contribute to the elastic energy gained by the element, the following virtual work equivalence holds true:

$\boldsymbol{q}_{\mathrm{int}} \cdot \delta \boldsymbol{a}=\overline{\boldsymbol{q}}_{\mathrm{int}} \cdot \delta \overline{\boldsymbol{a}}$, defining the internal-force vector $\boldsymbol{q}_{\text {int }}$ work-conjugated to the overall parameters $\boldsymbol{a}$. The linearization of the virtual work equivalence (A.9) yields:

$\Delta\left(\boldsymbol{q}_{\mathrm{int}} \cdot \delta \boldsymbol{a}\right)=\Delta\left(\overline{\boldsymbol{q}}_{\mathrm{int}} \cdot \delta \overline{\boldsymbol{a}}\right)$.

Introducing the consistent tangent stiffness tensor and its core-element counterpart (denoted as $\boldsymbol{K}_{\boldsymbol{q}_{\mathrm{int}}^{e}}$ in equation (44)), respectively:

$\boldsymbol{K}_{\mathrm{int}}=\frac{\partial \boldsymbol{q}_{\mathrm{int}}}{\partial \boldsymbol{a}}, \quad \overline{\boldsymbol{K}}_{\mathrm{int}}=\frac{\partial \overline{\boldsymbol{q}}_{\mathrm{int}}}{\partial \overline{\boldsymbol{a}}}$,

and recalling that $\Delta \delta \boldsymbol{a}=\mathbf{0}$, equation (A.10) can be developed in:

$\boldsymbol{K}_{\mathrm{int}} \Delta \boldsymbol{a} \cdot \delta \boldsymbol{a}=\overline{\boldsymbol{K}}_{\mathrm{int}} \Delta \overline{\boldsymbol{a}} \cdot \delta \overline{\boldsymbol{a}}+\overline{\boldsymbol{q}}_{\mathrm{int}} \cdot \Delta \delta \overline{\boldsymbol{a}}$.

In order to obtain the overall counterparts $\boldsymbol{q}_{\text {int }}$ and $\boldsymbol{K}_{\text {int }}$ of the core-element quantities $\overline{\boldsymbol{q}}_{\text {int }}$ and $\overline{\boldsymbol{K}}_{\text {int }}$, the crucial ingredient is the so-called projector operator $\boldsymbol{\Pi}$, able to extract the deformational part from incremental displacements:

$\delta \bar{a}=\Pi \delta \boldsymbol{a}$.

In fact, substituting (A.13) in (A.9) it turns out that

$\boldsymbol{q}_{\mathrm{int}}=\boldsymbol{\Pi}^{\mathrm{T}} \overline{\boldsymbol{q}}_{\mathrm{int}}$.

Analogously, the substitution of equation (A.13) in (A.12), yields:

$\boldsymbol{K}_{\mathrm{int}}=\boldsymbol{\Pi}^{\mathrm{T}} \overline{\boldsymbol{K}}_{\mathrm{int}} \boldsymbol{\Pi}+\boldsymbol{K}_{\boldsymbol{\Pi}^{\mathrm{T}}}\left[\overline{\boldsymbol{q}}_{\mathrm{int}}\right]$,

where:

$\boldsymbol{K}_{\boldsymbol{\Pi} \mathrm{T}}[\boldsymbol{v}] \Delta \boldsymbol{a}=\left.\Delta\left(\boldsymbol{\Pi}^{\mathrm{T}} \boldsymbol{v}\right)\right|_{\boldsymbol{v}=\mathrm{const}}$

The latter represents a geometric stiffness arising from the dependence of the projector operator from the nodal parameters $\boldsymbol{a}$. In fact, from (A.8), the projector operator depends on the rigid motion $r$ (A.3), which in turn depends on the nodal parameters $\boldsymbol{a}$. In particular, in order for the corotational approach to be effective, the rotation tensor $\boldsymbol{R}$ characterising $r$ must be chosen such that $\bar{f}$ is free of large rigid rotations. In the present work, $\boldsymbol{R}$ is identified with the rotation tensor of the polar decomposition of the gradient of the homogeneous transformation mapping the element nodes $V_{i}$ into their current positions $V_{i}^{\prime}=V_{i}+\boldsymbol{u}_{i}$ [13]. The reference point $G$ and the translation vector $t$ are, respectively, the element centroid and the mean of the nodal displacements $\boldsymbol{u}_{i}$ [25]. In particular, such choices ensure conditions (49) to be satisfied. Further details, including the derivation of $\boldsymbol{\Pi}$ and $\boldsymbol{K}_{\boldsymbol{\Pi}}{ }^{\mathrm{T}}$ are provided in references $[7,8]$.

\section{B Coated ellipsoid in uniform electric field}

The problem of an ellipsoidal particle with semidiameters $a>$ $b>c$, coated with a membrane having finite admittance, and embedded in a uniform electric field $\boldsymbol{E}_{\infty}$ established in the infinite space, is here considered.

Let $\{O ; x, y, z\}$ be a Cartesian reference system having the origin $O$ at the ellipsoid center and the coordinate axes parallel to the ellipsoid axes. The problem at hand is conveniently treated using an ellipsoidal coordinate system $\{\xi, \zeta, \eta\}$ 
(e.g., see [46]). In particular, the parameter $\xi$ describes a family of confocal ellipsoidal surfaces:

$\frac{z^{2}}{a^{2}+\xi}+\frac{x^{2}}{b^{2}+\xi}+\frac{y^{2}}{c^{2}+\xi}=1$,

and $\xi=0$ is the particle boundary. In the notation of Section 2, the ellipsoid and the outer media respectively correspond to the regions $\Omega_{1}$ and $\Omega_{2}$, with complex conductivities $\sigma_{1}^{*}$ and $\sigma_{2}^{*}$, whereas the particle surface coincides with the two-dimensional interface $\Gamma$, with admittance $Y$ per unit area. For the problem to have a simple analytical solution, $Y$ is assumed to vary over $\Gamma$ according to the equation:

$Y=Y_{0} \frac{h_{0}}{h}$,

where $Y_{0}$ is the average admittance per unit area over $\Gamma$ and:

$h=\frac{1}{2} \frac{\sqrt{(\xi-\eta)(\xi-\zeta)}}{\sqrt{\left(a^{2}+\xi\right)\left(b^{2}+\xi\right)\left(c^{2}+\xi\right)}}$,

$h_{0}=\left(\frac{1}{|\Gamma|} \int_{\Gamma} \frac{1}{h} \mathrm{~d} A\right)^{-1}$,

with $|\Gamma|$ denoting the measure of $\Gamma$. In passing, it is observed that $h$ is one of the metric coefficients associated to the ellipsoidal coordinate system [46]. A similar problem, concerning an ellipsoidal particle coated with a shell of non-uniform thickness, is considered in [3].

The electric field $\boldsymbol{E}_{\infty}$ can be assumed, without loss of generality, to be parallel to the $z$-axis, with intensity $E_{\infty}$ (the other two components can be treated analogously, and the linearity of the problem allows to sum the contributions $[46,3])$. Accordingly, the resulting electric potential $\Psi$ solves the problem stated in equations (11)-(13), supplemented with the following boundary condition at infinity:

$\Psi=\Psi_{\infty}, \quad$ for $\xi \rightarrow+\infty$,

where the potential $\Psi_{\infty}$ is given by:

$\Psi_{\infty}=-E_{\infty} z=-E_{\infty} \frac{\sqrt{\left(a^{2}+\xi\right)\left(a^{2}+\eta\right)\left(a^{2}+\zeta\right)}}{\sqrt{\left(a^{2}-b^{2}\right)\left(a^{2}+c^{2}\right)}}$.

This problem can be solved in the same fashion explored in [46]. To this end, it is convenient to introduce the function:

$A(\xi)=\frac{a b c}{2} \int_{\xi}^{+\infty}\left[\left(a^{2}+s\right)^{3}\left(b^{2}+s\right)\left(c^{2}+s\right)\right]^{-\frac{1}{2}} \mathrm{~d} s$,

and the following quantities:

$c_{1}=\frac{\sigma_{1}^{*}-\sigma_{2}^{*}}{\sigma_{2}^{*}}, \quad c_{2}=\frac{1}{2} \frac{\sigma_{1}^{*}}{Y_{0} h_{0} a^{2}}$,

$c_{3}=c_{1}-c_{2}, \quad c_{4}=\left[1+c_{2}+c_{3} A(0)\right]^{-1}$.

Then, the potential $\Psi$ turns out to be:

$\Psi= \begin{cases}\Psi_{\infty} c_{4}, & \xi<0, \\ \Psi_{\infty}\left[1-c_{3} c_{4} A(\xi)\right], & \xi>0,\end{cases}$

and, consequently, its jump across $\Gamma$ is given by:

$\llbracket \Psi \rrbracket=\left.c_{2} c_{4} \Psi_{\infty}\right|_{\xi=0}$.

The electric field on both sides of $\Gamma$ is computed exploiting the decomposition (35). More specifically, the normal component $E_{\boldsymbol{n}}$ immediately follows from equations (36) and (A.25), whereas the tangential projection $\boldsymbol{E}_{\tau}$ is derived from equation (A.24). In particular, the latter computation is simplified by the fact that the tangential gradient on $\Gamma$ of any function of the only coordinate $\xi$ identically vanishes. Accordingly, the electric field on the ellipsoid surface $\Gamma$ takes the form:

$\boldsymbol{E}=\boldsymbol{E}_{\tau}+E_{\boldsymbol{n}} \boldsymbol{n}$

where:

$\boldsymbol{E}_{\tau}= \begin{cases}E_{\infty} c_{4} \boldsymbol{P}_{\tau} \boldsymbol{k}, & \xi<0, \\ E_{\infty}\left[1-c_{3} c_{4} A(0)\right] \boldsymbol{P}_{\tau} \boldsymbol{k}, & \xi>0,\end{cases}$

and $\boldsymbol{k}$ is the unit vector parallel to the $z$-axis. Finally, exploiting equations (10) and $(7)_{2}$, the surface forces $f_{\Gamma}$ induced by the electric field can be expressed as:

$\boldsymbol{f}_{\Gamma}=\boldsymbol{f}_{\tau}+f_{\boldsymbol{n}} \boldsymbol{n}$

where:

$\boldsymbol{f}_{\tau}=\frac{1}{4} \llbracket \varepsilon\left(\boldsymbol{E}_{\tau} \bar{E}_{\boldsymbol{n}}+\overline{\boldsymbol{E}}_{\tau} E_{\boldsymbol{n}}\right) \rrbracket$,

$f_{\boldsymbol{n}}=\frac{1}{4} \llbracket \varepsilon\left(\left|E_{\boldsymbol{n}}\right|^{2}-\left\|\boldsymbol{E}_{\tau}\right\|^{2}\right) \rrbracket$,

respectively represent the tangential projection and the normal component of $\boldsymbol{f}_{\Gamma}$.

\section{References}

1. Ahmad, I.L., Ahmad, M.R.: Trends in characterizing single cell's stiffness properties. Micro Nano Syst Lett 2(8) (2014). DOI 10.1186/s40486-014-0008-5

2. Asami, K.: Effectiveness of "thin-layer" and "effective medium" approximations in numerical simulation of dielectric spectra of biological cell suspensions. Jpn J Appl Phys 49(12R), 127,001 (2010). DOI 10.1143/JJAP.49.127001

3. Asami, K., Hanai, T., Koizumi, N.: Dielectric approach to suspensions of ellipsoidal particles covered with a shell in particular reference to biological cells. Jpn J Appl Phys 19(2), 359-365 (1980). DOI 10.1143/JJAP.19.359

4. Bisegna, P., Caselli, F.: A simple formula for the effective complex conductivity of periodic fibrous composites with interfacial impedance and applications to biological tissues. J Phys D: Appl Phys 41, 115,506 (2008). DOI 10.1088/0022-3727/41/11/115506

5. Boey, S.K., Boal, D.H., Discher, D.E.: Simulations of the erythrocyte cytoskeleton at large deformation. I. Microscopic models. Biophys J 75(3), 1573-1583 (1998). DOI 10.1016/S0006-3495(98)74075-5

6. Bustamante, C., Bryant, Z., Smith, S.B.: Ten years of tension: single-molecule DNA mechanics. Nature 421, 423-427 (2003). DOI 10.1038/nature01405

7. Caselli, F., Bisegna, P.: Polar decomposition based corotational framework for triangular shell elements with distributed loads. Int J Numer Methods Eng 95(6), 499-528 (2013). DOI 10.1002/nme.4528

8. Caselli, F., Bisegna, P.: A corotational flat triangular element for large strain analysis of thin shells with application to soft biological tissues. Comput Mech 54(3), 847-864 (2014). DOI 10.1007/s00466-014-1038-9 
9. Caselli, F., Bisegna, P., Maceri, F.: EIT-inspired microfluidic cytometer for single-cell dielectric spectroscopy. J Microelectromech Syst 19(5), 1029-1040 (2010). DOI 10.1109/JMEMS.2010.2067204

10. Caselli, F., Shaker, M., Colella, L., Renaud, P., Bisegna, P.: Modeling, simulation, and performance evaluation of a novel microfluidic impedance cytometer for morphology-based cell discrimination. $\mathrm{J} \mathrm{Mi-}$ croelectromech Syst 23(4), 785-794 (2014). DOI 10.1109/JMEMS.2014.2325979

11. Chen, J., Abdelgawad, M., Yu, L., Shakiba, N., Chien, W.Y., Lu, Z., Geddie, W.R., Jewett, M.A.S., Sun, Y.: Electrodeformation for single cell mechanical characterization. J Micromech Microeng 21, 054,012 (2011). DOI 10.1088/0960-1317/21/5/054012

12. Chiabrera, A., Nicolini, C.A., Schwan, H.P. (eds.): Interactions between electromagnetic fields and cells. NATO ASI. Series A: Life sciences. Plenum Press, New York (1985)

13. Crisfield, M.A.: Non-linear Finite Element Analysis of Solids and Structures, vol. 2: Advanced Topics. John Wiley \& Sons Ltd, Chichester (1997)

14. Dao, M., Li, J., Suresh, S.: Molecularly based analysis of deformation of spectrin network and human erythrocyte. Mater Sci Eng C-Mater Biol Appl 26(8), 1232-1244 (2006). DOI 10.1016/j.msec.2005.08.020

15. Dao, M., Lim, C.T., Suresh, S.: Mechanics of the human red blood cell deformed by optical tweezers. J Mech Phys Solids 51(11-12), 2259-2280 (2003). DOI 10.1016/j.jmps.2003.09.019

16. Di Carlo, D.: A mechanical biomarker of cell state in medicine. J Lab Autom 17(1), 32-42 (2012). DOI $10.1177 / 2211068211431630$

17. Discher, D.E., Boal, D.H., Boey, S.K.: Phase transitions and anisotropic responses of planar triangular nets under large deformation. Phys Rev E 55(4), 4762-4772 (1997). DOI 10.1103/PhysRevE.55.4762

18. Discher, D.E., Boal, D.H., Boey, S.K.: Simulations of the erythrocyte cytoskeleton at large deformation. II. Micropipette aspiration. Biophys J 75(3), 1584-1597 (1998). DOI 10.1016/S0006-3495(98)74076-7

19. Do Carmo, M.P.: Differential geometry of curves and surfaces. Prentice Hall, Englewood Cliffs, NJ (1976)

20. Doh, I., Lee, W.C., Cho, Y.H., Pisano, A.P., Kuypers, F.A.: Deformation measurement of individual cells in large populations using a single-cell microchamber array chip. Appl Phys Lett 100(17) (2012). DOI 10.1063/1.4704923

21. Du, E., Dao, M., Suresh, S.: Quantitative biomechanics of healthy and diseased human red blood cells using dielectrophoresis in a microfluidic system. Extr Mech Lett 1, 35-41 (2014). DOI 10.1016/j.eml.2014.11.006

22. Evans, E., Fung, Y.C.: Improved measurements of erythrocyte geometry. Microvasc Res 4(4), 335-347 (1972). DOI 10.1016/0026-2862(72)90069-6

23. Fedosov, D.: Multiscale modeling of blood flow and soft matter. Ph.D. thesis, Division of Applied Mathematics, Brown University, USA (2010)

24. Fedosov, D.A., Caswell, B., Karniadakis, G.E.: A multiscale red blood cell model with accurate mechanics, rheology, and dynamics. Biophys J 98(10), 2215-2225 (2010). DOI 10.1016/j.bpj.2010.02.002

25. Felippa, C.A., Haugen, B.: A unified formulation of smallstrain corotational finite elements: I. Theory. Comput Meth Appl Mech Eng 194(21-24), 2285-2335 (2005). DOI 10.1016/j.cma.2004.07.035
26. Foster, K.R., Schwan, H.P.: Dielectric properties of tissues and biological materials: a critical review. Crit Rev Biomed Eng 17(2), 25-104 (1989)

27. Gawad, S., Cheung, K., Seger, U., Bertsch, A., Renaud, P.: Dielectric spectroscopy in a micromachined flow cytometer: theoretical and practical considerations. Lab Chip 4, 241-251 (2004). DOI 10.1039/b313761a

28. Gossett, D.R., Tse, H.T.K., Lee, S.A., Ying, Y., Lindgren, A.G., Yang, O.O., Rao, J., Clark, A.T., Di Carlo, D.: Hydrodynamic stretching of single cells for large population mechanical phenotyping. Proc Natl Acad Sci 109(20), 7630-7635 (2012). DOI 10.1073/pnas.1200107109

29. Guido, I., Jaeger, M.S., Duschl, C.: Dielectrophoretic stretching of cells allows for characterization of their mechanical properties. Eur Biophys J 40(3), 281-8 (2011). DOI 10.1007/s00249-010-0646-3

30. Haque, M.M.: Elastic theory for the deformation of a spherical dielectric biological object under electro-optical trapping. RSC Adv 5(55), 44,458-44,462 (2015). DOI 10.1039/C5RA06125C

31. Helfrich, W.: Elastic properties of lipid bilayers: theory and possible experiments. Z Naturforsch C 28(11), 693703 (1973)

32. Kim, D.H., Wong, P.K., Park, J., Levchenko, A., Sun, Y.: Microengineered platforms for cell mechanobiology. Annu Rev Biomed Eng 11, 203-233 (2009). DOI 10.1146/annurev-bioeng-061008-124915

33. Kirsch, A.: The domain derivative and two applications in inverse scattering theory. Inverse Prob 9(1), 81-96 (1999). DOI 10.1088/0266-5611/9/1/005

34. Landau, L.D., Lifshitz, E.M.: Electrodynamics of Continuous Media, Course of Theoretical Physics, vol. 8, second edn. Pergamon, Amsterdam (1984)

35. Lee, J.C.M., Wong, D.T., Discher, D.E.: Direct measures of large, anisotropic strains in deformation of the erythrocyte cytoskeleton. Biophys J 77(2), 853-864 (1999). DOI 10.1016/S0006-3495(99)76937-7

36. Leung, S.L., Lu, Y., Bluestein, D., Slepian, M.J.: Dielectrophoresis-mediated electrodeformation as a means of determining individual platelet stiffness. Ann Biomed Eng pp. 1-11 (2015). DOI 10.1007/s10439-0151383-7

37. Li, J., Dao, M., Lim, C.T., Suresh, S.: Spectrin-level modeling of the cytoskeleton and optical tweezers stretching of the erythrocyte. Biophys J 88(5), 3707-3719 (2005). DOI 10.1529/biophysj.104.047332

38. Lim, C.T., Dao, M., Suresh, S., Sow, C.H., Chew, K.T.: Corrigendum to "Large deformation of living cells using laser traps" [Acta Mat 52(7), 1837-1845 (2004)]. Acta Mat 52(13), 4065-4066 (2004). DOI 10.1016/j.actamat.2004.05.016

39. Lim, C.T., Dao, M., Suresh, S., Sow, C.H., Chew, K.T.: Large deformation of living cells using laser traps. Acta Mat 52(7), 1837-1845 (2004). DOI 10.1016/j.actamat.2004.05.016

40. MacQueen, L.A., Buschmann, M.D., Wertheimer, M.R.: Mechanical properties of mammalian cells in suspension measured by electro-deformation. J Micromech Microeng 20(6), 065,007 (2010). DOI 10.1088/0960$1317 / 20 / 6 / 065007$

41. Marko, J.F., Siggia, E.D.: Stretching DNA. Macromolecules 28(26), 8759-8770 (1995). DOI $10.1021 / \mathrm{ma00130a008}$

42. Mills, J.P., Qie, L., Dao, M., Lim, C.T., Suresh, S.: Nonlinear elastic and viscoelastic deformation of the human 
red blood cell with optical tweezers. Mech Chem Biosyst 1(3), 169-180 (2004)

43. Oñate, E., Zárate, F.: Rotation-free triangular plate and shell elements. Int $J$ Numer Methods Eng 47(1-3), 557-603 (2000). DOI 10.1002/(SICI)10970207(20000110/30)47:1/3;557::AID-NME784¿3.0.CO;2-9

44. Rodriguez, M.L., McGarry, P.J., Sniadecki, N.J.: Review on cell mechanics: Experimental and modeling approaches. Appl Mech Rev 65(6), 060,801 (2013). DOI $10.1115 / 1.4025355$

45. Somersalo, E., Cheney, M., Isaacson, D.: Existence and uniqueness for electrode models for electric current computed tomography. SIAM J Appl Math 52, 1023-1040 (1992). DOI 10.1137/0152060

46. Stratton, J.A.: Electromagnetic Theory. McGraw-Hill (1941)

47. Sukhorukov, V.L., Mussauer, H., Zimmermann, U.: The effect of electrical deformation forces on the electropermeabilization of erythrocyte membranes in low- and high-conductivity media. J Membr Biol 163(3), 235-245 (1998). DOI 10.1007/s002329900387

48. Turner, M.J., Clough, R.W., Martin, H.C., Topp, L.J.: Stiffness and deflection analysis of complex structures. J Aeronaut Sci 23(9), 805-824 (1956)

49. Vlahovska, M.P., Gracià, R.S., Aranda-Espinoza, S., Dimova, R.: Electrohydrodynamic model of vesicle deformation in alternating electric fields. Biophys J 96(12), 4789-4803 (2009). DOI 10.1016/j.bpj.2009.03.054

50. Voldman, J.: Electrical forces for microscale cell manipulation. Annu Rev Biomed Eng 8(1), 425-454 (2006). DOI 10.1146/annurev.bioeng.8.061505.095739

51. Wang, X., Wang, X.B., Gascoyne, P.R.C.: General expressions for dielectrophoretic force and electrorotational torque derived using the Maxwell stress tensor method. J Electrostat 39(4), 277-295 (1997). DOI 10.1016/S03043886(97)00126-5

52. Wong, P.K., Tan, W., Ho, C.M.: Cell relaxation after electrodeformation: effect of latrunculin a on cytoskeletal actin. J Biomech 38(3), 529-535 (2005). DOI 10.1016/j.jbiomech.2004.04.008

53. Yan, W.J., Ma, Y.C.: The application of domain derivative for heat conduction with mixed condition in shape reconstruction. Appl Math Comput 181(2), 894-902 (2006). DOI 10.1016/j.amc.2006.02.011

54. Zheng, Y., Nguyen, J., Wei, Y., Sun, Y.: Recent advances in microfluidic techniques for single-cell biophysical characterization. Lab Chip 13, 2464-2483 (2013). DOI $10.1039 / \mathrm{c} 31 \mathrm{lc} 50355 \mathrm{k}$

55. Zhou, Y.X., Sze, K.Y.: A geometric nonlinear rotationfree triangle and its application to drape simulation. Int J Numer Methods Eng 89(4), 509-536 (2012). DOI 10.1002/nme.3250 